\title{
The low-luminosity tail of the GRB distribution: The case of GRB $980425\left(^{*}\right)$
}

\author{
F. Daigne $\left({ }^{1}\right)\left({ }^{2}\right)$ and R. Mochkovitch $\left({ }^{1}\right)$ \\ $\left({ }^{1}\right)$ Institut d'Astrophysique de Paris - 98 bis boulevard Arago, 75014 Paris, France \\ $\left(^{2}\right)$ Université Pierre et Marie Curie - Paris VI - 4 place Jussieu, 75005 Paris, France
}

(ricevuto il 23 Maggio 2005; pubblicato online il 20 Settembre 2005)

\begin{abstract}
Summary. - The association of GRB 980425 with the nearby supernova SN $1998 \mathrm{bw}$ at $z=0.0085$ implies the existence of a population of GRBs with an isotropic-equivalent luminosity which is about $10^{4}$ times smaller than in classical cosmic GRBs. We investigate two scenarios to explain the peculiar properties of GRB 980425: a normal (intrinsically bright) GRB seen off-axis or an intrinsically weak GRB seen on-axis.
\end{abstract}

PACS 98.70.Rz - $\gamma$-ray sources; $\gamma$-ray bursts.

PACS 97.60. Bw - Supernovae.

PACS 01.30.Cc - Conference proceedings.

\section{1. - Introduction: the association of GRB 980425 with SN 1998bw}

Beppo-SAX observations of GRB 980425 show that it is a standard long-duration $(\sim 31 \mathrm{~s})$ single pulse burst with a rather low but still not uncommon peak energy $E_{\mathrm{p}}=$ $68 \pm 40 \mathrm{keV}$ [4]. However if the association of GRB 980425 with its proposed counterpart SN 1998bw [5] is real, the observed gamma-ray peak flux $\left(\sim 2.4 \times 10^{-7} \mathrm{erg} \mathrm{cm}^{-2} \mathrm{~s}^{-1}\right)$ corresponds to an unusual low isotropic equivalent luminosity $L_{\gamma, 4 \pi} \sim 3 \times 10^{46} \mathrm{erg} \mathrm{s}^{-1}$, as the redshift of SN 1998bw $(z=0.008)$ is notably smaller than all the other measured GRB redshifts $(0.105 \leq z \leq 4.5)$. We assume in the following that a GRB is produced by relativistic ejecta of Lorentz factor $\Gamma$ and opening angle $\Delta \theta \gg 1 / \Gamma$ generated by a source at redshift $z$. We define $\theta_{0}$ as the angle between the line-of-sight and the axis of the ejecta. The observed bolometric peak flux and peak energy are given by

$$
P^{\mathrm{obs}}=\frac{L_{\mathrm{rad}, 4 \pi}}{4 \pi D_{\mathrm{L}}^{2}} \quad \text { and } \quad E_{p}^{\mathrm{obs}}=\frac{E_{p}}{1+z}
$$

(*) Paper presented at the "4th Workshop on Gamma-Ray Burst in the Afterglow Era", Rome, October 18-22, 2004.

(C) Società Italiana di Fisica 


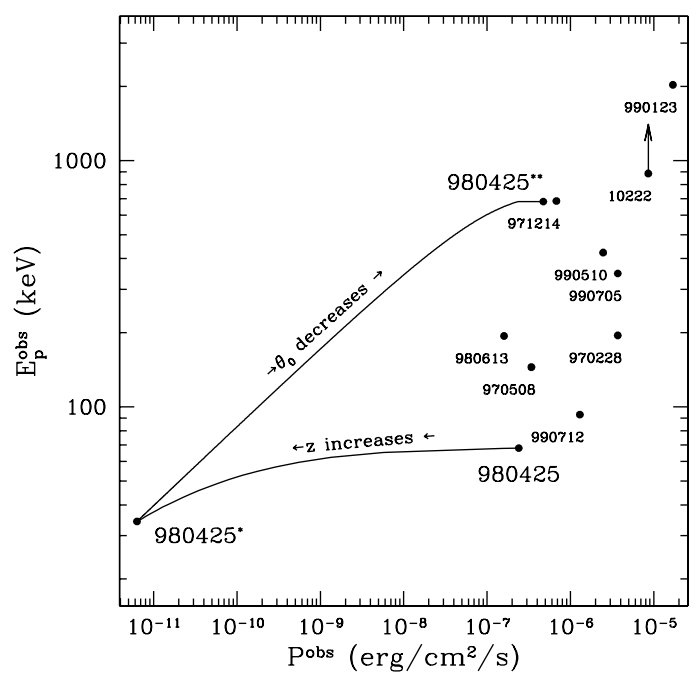

Fig. 1. - GRB 980425 as a bright GRB seen off-axis.

in the on-axis case and

$$
P^{\mathrm{obs}} \simeq \frac{1}{2\left(1+\Gamma^{2}\left(\theta_{0}-\Delta \theta\right)^{2}\right)^{3}} \frac{L_{\mathrm{rad}, 4 \pi}}{4 \pi D_{\mathrm{L}}^{2}} \quad \text { and } \quad E_{p}^{\mathrm{obs}} \simeq \frac{1}{1+\Gamma^{2}\left(\theta_{0}-\Delta \theta\right)^{2}} \frac{E_{p}}{1+z},
$$

in the off-axis case, where the intrinsic parameters of the GRB in the source frame are the isotropic equivalent luminosity $L_{\mathrm{rad}, 4 \pi}$ and the peak energy $E_{\mathrm{p}}$. Therefore, the peculiar properties of GRB 980425 may have two origins: i) either this burst is only apparently weak due to a large viewing angle but has normal intrinsic properties, with $L_{\mathrm{rad}, 4 \pi} \geq$ a few $10^{50} \mathrm{erg} \mathrm{s}^{-1}$; ii) or it is intrinsically weak with $L_{\mathrm{rad}, 4 \pi} \simeq 3 \times 10^{46} \mathrm{erg} \mathrm{s}^{-1}$ and is observed on-axis. In the following, we successively investigate these two scenarios. In the second case, the intrinsic properties of GRBs are studied in the framework of the internal shock model $[9,2]$.

\section{2. - Low-luminosity GRBs as normal GRBs seen off-axis}

2`1. Effect of a low redshift and a large viewing angle. - We have plotted in fig. 1 the location of GRB 980425 as well as the other Beppo-SAX GRBs with a known redshift in a bolometric peak flux-peak energy diagram. We move GRB 980425 from $z=0.008$ to $z=1$. The final point is named GRB $980425^{*}$. It is clearly much too weak to be observed as a GRB or even an XRF. We then move GRB $980425^{*}$ up to $\theta_{0}=0$ (on-axis observation), assuming that GRB 980425 is seen off-axis with $\theta_{0}=\Delta \theta+k / \Gamma$ (here $\left.k=4\right)$. The final point is named GRB $980425^{* *}$ and is clearly back in the GRB region, very close to GRB 971214. We conclude that the observed properties of GRB 980425 are compatible with those of an intrinsically bright GRB observed off-axis, as proposed by [13].

2. 2 . A statistical problem. - The local apparent GRB rate is very low: $\sim 1 /(20000 \mathrm{yr})$ within $40 \mathrm{Mpc}[8,11,7,6]$. Then the detection of GRB 980425 at $z=0.008$ (distance $\sim 34 \mathrm{Mpc}$ ) means that i) either we have observed by chance a very rare event, ii) or the local apparent rate of GRB 980425-like events is much higher than the standard GRB rate. The ratio of the rates of off-axis over on-axis GRB observations within $40 \mathrm{Mpc}$ 


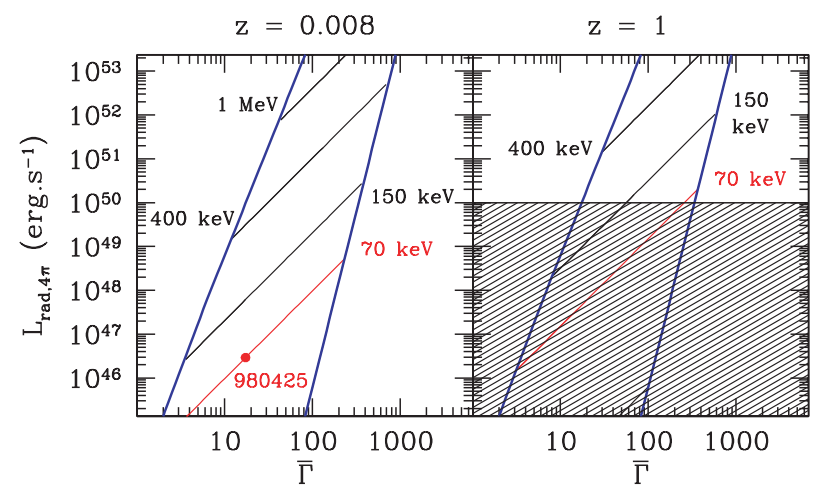

Fig. 2. - The parameter space of the internal shock model: in the radiated luminosity-Lorentz factor plane, lines of GRBs at constant peak energy are plotted for local GRBs (left panel) and "standard" cosmological GRBs (right panel). The constant peak-energy lines are limited by two thick lines corresponding to the "transparency" (left side) and "deceleration" constraints (see text). The region which is not observable according to the typical sensitivity of GRB detectors is indicated by a shaded area. The location of GRB 980425 is indicated by a big dot.

is dominated by the intrinsic ratio of the solid angle covered by the emission in this two cases: Rate (off-axis)/Rate (on-axis) $\simeq(\Omega / 4 \pi)_{\text {off-axis }} /(\Omega / 4 \pi)_{\text {on-axis. }}$ For off-axis observations, the flux becomes negligible as soon as $\theta_{0}-\Delta \theta \gg 1 / \Gamma$. Therefore, if the typical opening angle $\Delta \theta$ of the ejecta is large compared to $1 / \Gamma$, off-axis events should be even rarer events than on-axis GRBs. It is only if $\Delta \theta \ll 1 / \Gamma$ that the opposite situation can be expected. Such a geometry is clearly not favored by models of the central engine.

\section{3. - Low-luminosity GRBs as intrinsically weak GRBs}

In this section, we assume that the prompt gamma-ray emission from GRBs is dominated by the radiation of electrons accelerated by internal shocks within the relativistic ejecta. The corresponding intrinsic luminosity and peak energy of the burst are given by $L_{\mathrm{rad}, 4 \pi} \simeq \alpha_{\mathrm{e}} \times f_{\mathrm{d}}(\kappa) \times L_{\mathrm{kin}}$ and $E_{\mathrm{p}} \simeq 2 \Gamma \times E_{\mathrm{p}}^{\mathrm{com}} \simeq K L_{\mathrm{kin}}^{x} \Phi_{x y}(\kappa) \Gamma^{-(6 x-1)} \tau^{-2 x}$, where $L_{\text {kin }}$ is the initial kinetic energy flux in the ejecta, $\Gamma$ is its Lorentz factor, $\kappa$ is the typical ratio $\Gamma_{\max } / \Gamma_{\min }$ characterizing the variation of the Lorentz factor within the ejecta and $\tau$ the timescale of the ejection, typically comparable with the observed duration, $f_{\mathrm{d}}$ is the efficiency of the kinetic-to-internal energy conversion within internal shocks, $\alpha_{\mathrm{e}}$ is the fraction of the dissipated energy which is injected in the population of non-thermal electrons, $K, x$ and $y$ are parameters related to the dominant emission process and $\Phi_{x y}$ is a steadily increasing function of $\kappa$. For the standard synchrotron process, $K$ depends only on physical constants and $x=1 / 2, y=5 / 2$. However, the properties of pulses within the lightcurves of GRBs favor smaller values of $x$ and $y$ [3]. As the details of the microphysics within the shocked material are poorly understood and well beyond the scope of this study, we fix $x=y=1 / 4$ (such exponents can be obtained in the standard synchrotron process if the equipartition parameters depend on the shock intensity), $\alpha_{\mathrm{e}}=0.5$ and we adjust $K$ so that a "typical" GRB at $z=1$ with $L_{\mathrm{kin}}=10^{52} \mathrm{erg} \mathrm{s}^{-1}, \tau=10 \mathrm{~s}, \Gamma=300$ and $\kappa=4$ has an observed peak energy of $200 \mathrm{keV}$. We then focus on the parameter space which is now limited to four parameters: $L_{\text {kin }}$, $\Gamma, \kappa$ and $\tau$. Several constraints apply: 1) internal shocks cannot happen too close to the central source as the ejecta must be already transparent; 2) the ejecta has also to be transparent to pairs during the internal shock phase to avoid an unobserved cutoff 
in the high energy spectrum; 3) internal shocks must start before the deceleration by the external medium becomes efficient, otherwise they can be suppressed by the reverse shock. In fig. 2, we have plotted in thick lines the location of these constraints in a $L_{\text {rad }}-\Gamma$ plane, for $\kappa=4$ and $\tau=30 \mathrm{~s}$, which are realistic values for GRB 980425 . The external medium has a constant density $n=1 \mathrm{~cm}^{-3}$. We have also plotted lines of GRBs with constant observed peak energy, for $z=0.008$ (left panel) and $z=1$ (right panel). Finally, we have indicated with a shaded area the GRBs which are below the current instrument sensitivity.

Figure 2 shows clearly that a large diversity of GRBs is expected in this scenario. It includes GRB 980425, which is indicated by a big dot: it appears as an intrinsically weak and soft GRB with a low injected power in the wind $\left(L_{\mathrm{kin}} \sim 3 \times 10^{47} \mathrm{erg} \mathrm{s}^{-1}\right)$ and a low Lorentz factor $(\bar{\Gamma} \sim 20-30)$. Such parameters could correspond to an event with an unefficient central engine. Due to this intrinsically low luminosity, it is clear that such an event can be detected at low redhshift only (in the $z=1$ part of the figure, it is clearly below the detection threshold).

\section{4. - Diversity of the GRB population in the internal shock model}

The internal shock model does not predict the distribution of the parameters $L_{\text {kin }}$, $\Gamma, \kappa$ and $\tau$, as they are more related to the poorly understood physics of the central engine. If we assume that the initial event responsible for a GRB is a collapsar, the differences between the observed rates of bright and weak GRBs can be interpreted in this scenario as follows: i) only a small fraction of collapsars is able to produce a highly relativistic $(\Gamma \geq 100)$ and highly energetic $\left(L_{\mathrm{kin}} \geq 10^{50} \mathrm{erg} \mathrm{s}^{-1}\right)$ outflow. These events are responsible for GRBs (as well as X-ray rich GRBs and XRFs, the difference between these sub-categories coming from other parameters such as the variability in the Lorentz factor, see [1]). All these events are rare but can be detected at high redshift so that the observed rate is high. They have normal afterglows. ii) A larger but still small fraction of collapsars leads to mildly relativistic, mildly energetic outflows. They produce GRB 980425-like events. As they can be detected only in the local Universe, the apparent rate is small. They should have weak afterglows too (see [12]). GRB 031203 [10] can appear as an intermediate case between normal GRBs and GRB 980425. iii) Most collapsars do not produce relativistic outflows. Either the star collapses directly to a black hole or gives a "normal" type-Ic supernova.

\section{REFERENCES}

[1] Barraud C., Daigne F., Atteia J.L. and Mochkovitch R., to be published in $A \mathscr{E} A$, (2005).

[2] Daigne F. and Mochkovitch R., MNRAS, 296 (1998) 275.

[3] Daigne F. and Mochkovitch R., MNRAS, 342 (2003) 587.

[4] Frontera F. et al., ApJS, 127 (2000) 59.

[5] Galama T.J. et al., Nature, 395 (1998) 670.

[6] Guetta D. et al., APJ, 615 (2004) L73.

[7] Perna R. et al., APJ, 594 (2003) 379.

[8] Porciani C. and Madau P., ApJ, 548 (2001) 522.

[9] Rees M. and Mészáros P., APJ, 430 (1994) L93.

[10] Sazonov S. and Lutovinoc A. and Sunyaev, R., Nature, 430 (2004) 646.

[11] Schmidt M., APJ, 552 (2001) 36.

[12] Soderberg A.M. et al., Nature, 430 (2004) 648.

[13] Yamazaki R., Yonetoku D. and Nakamura T., ApJ, 594 (2003) L79. 